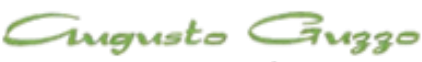

REVISTA ACADEMICA

\title{
Uma Análise da Gestão de Risco de Crédito nas Instituições Financeiras Brasileiras de Capital Aberto
}

\author{
Alexandre Rogério da Silva ${ }^{1}$ \\ Recebido em: 06/07/2013. Aprovado em: 16/09/2013. Disponibilizado em: 20/12/2013
}

1. Universidade Presbiteriana Mackenzie - CCSA/UPM São Paulo, SP, Brasil. e-mail: alerog@uol.com.br

\section{Resumo}

Com este artigo busca-se analisar as estratégias desenvolvidas pelas instituições financeiras brasileiras para a gestão de risco de crédito. A partir de um estudo detalhado das estratégias de gestão de risco de crédito, o artigo procura verificar se as instituições financeiras brasileiras fazem uso de modelos de risco de crédito, estando assim protegidas diante de um cenário de crise. O estudo alcançou seus resultados na medida em que estabeleceu um comparativo entre o que a literatura de gerenciamento de risco de crédito propõe como as instituições financeiras brasileiras praticam. Para a obtenção de informações relativa às empresas, fez-se uso do método qualitativoexploratório. Os resultados demonstraram que as Instituições Financeiras Brasileiras, fazem uso de modelos de Credit scoring e Credit Rating, que de acordo com Gestel et al (2009) é uma técnica eficiente de gestão de risco do tomador do empréstimo.

Palavras-chave: gestão de risco de crédito, credit rating, credit scoring, instituições financeiras brasileiras.

\begin{abstract}
With this article we try to analyze the strategies developed by Brazilian financial institutions to manage credit risk. From a detailed study of strategies for managing credit risk, the paper attempts to verify that Brazilian financial institutions make use of credit risk models, thus being protected against a background of crisis. The study achieved its results in that it established a comparison between what the literature of credit risk management proposes to Brazilian financial institutions practice. To obtain information on the companies made use of the qualitative exploratory method. The results showed that the Brazilian Financial Institutions make use of credit scoring models and Credit Rating, which according Gestel et al (2009) is an efficient technique for risk management of the borrower.
\end{abstract}

Keywords: management of credit risk, credit rating, credit scoring, Brazilian financial institutions 


\section{Introdução}

A crise financeira que assolou o mercado financeiro americano, no período de 2008 e 2009, trouxe grande instabilidade aos mercados financeiros mundiais, levando a falência centenas de Instituições Financeiras nos Estados Unidos. Segundo dados do FDIC (Federal Deposit Insurance Corporation) 454 bancos decretaram falência desde 2008, quando a crise teve inicio.

Neste novo cenário econômico estabelecido após o ano de 2008, a gestão de risco tem apresentado uma importância cada vez maior, não só para as Instituições Financeiras. A gestão do risco tem se apresentando como uma importante ferramenta para o aumento da competitividade, ajudando a preservar o resultado financeiro. $O$ risco é a probabilidade e a consequência de ocorrer determinado evento. (Kaplan e Garrick, 1981). Risco também pode ser definido como a variância da distribuição dos resultados (Dhar e Balakrishnan, 2006).

A atividade de gerenciamento de risco pode ser descrita como um processo de identificação, quantificação e desenvolvimento de estratégias para gerir o risco, com o objetivo de evitar perdas financeiras e diminuir a volatilidade dos lucros (Gestel e Baesens 2009). Existem diversos tipos de riscos na literatura, dos quais é possível destacar o Risco de mercado, Risco de liquidez, Risco operacional e Risco de crédito.

As Instituições financeiras têm como característica oferecer empréstimos para diversos agentes econômicos, como pessoas físicas e jurídicas, ficando assim expostas a diversos tipos de riscos, principalmente ao risco de crédito. No que se refere a perdas com crédito, segundo a agência de classificação de risco Austing Rating, os empréstimos atrasados no Brasil que os bancos registraram como prejuízo no primeiro semestre de 2012 totalizou $\mathrm{R} \$ 38$ bilhões de reais.

Dentre os diversos tipos de risco, este estudo pretende analisar o Risco de crédito e principalmente avaliar quais os modelos utilizados pelas instituições financeiras brasileiras para minimizar a exposição a esta categoria de risco.

A relevância deste estudo se da pelo crescente aumento da procura de crédito no Brasil. Segundo relatório do Banco Central, foram emprestados pelas instituições financeiras o valor de $\mathrm{R} \$ 2,237$ trilhões de reais para pessoas físicas e jurídicas. Além do aumento na procura de empréstimos a competitividade entre as empresas do setor faz com que as Instituições financeiras busquem por ferramentas que minimizem as perdas geradas pela inadimplência, buscando selecionar os clientes que possuem o melhor perfil de risco.

\section{Referencial teórico}

Risco pode ser definido como a probabilidade de um evento inesperado, que pode ou não acontecer. (Hansson, 2005). Indo ao encontro dessa afirmação, o risco financeiro é a probabilidade de uma empresa sofrer perdas financeiras devido a diversos fatores, como variações na taxa de juros, variação no cambio, variação no preço de commodity, desvalorização nos preços de títulos, etc. (Polak, Robertson e Lind, 2011).

Para diminuir os impactos causados pelo o acontecimento de eventos inesperados, tem crescido a utilização de ferramentas de gerenciamento de riscos das Instituições financeiras. A atividade de gerenciamento de risco é um processo de identificação, 
quantificação e desenvolvimento de estratégias para gerir o risco, com o objetivo de evitar perdas financeiras e diminuir a volatilidade dos lucros (Gestel e Baesens 2009).

A gestão de risco foi identificada em pesquisa como o "Objetivo mais importante para os executivos financeiros" (Froot, Scharfstein e Stein, 1993. p.1629, apud Rawls e Smithson 1990). Os autores desenvolveram um trabalho procurando analisar as políticas de gestão de risco corporativo, discutindo no artigo as estratégias de gerenciamento de risco por meio do hedge cambial para empresas multinacionais e estratégias com opções, os autores desenvolveram "um modelo simples das vantagens do hedge", o estudo chegou à conclusão que o gerenciamento de risco dentro de uma organização depende de diversos fatores, como por exemplo, a estratégia de Hedge ótimo vai depender da natureza da concorrência e das estratégias de hedge adotada pelos competidores.

Entretanto, de acordo com Stulz (1996) a evidência empírica vai de encontro com o explanado pela literatura, o autor afirma que a gestão de risco é limitada. No artigo publicado pelo autor em 1996, Stulz (1996) oferece uma nova abordagem que tenta ir ao encontro do mencionado pela literatura acadêmica. $\mathrm{O}$ autor ainda examina os impactos desta nova abordagem para a gestão da gestão de risco e para riscos de medidas como o VaR. O autor afirma que é possível uma empresa pode obter vantagens se expondo a certos tipos de risco, concluindo que ao ser detectada uma ineficiência no mercado, as pessoas responsáveis pelo o gerenciamento do risco devem sempre aproveitá-la, maximizando assim o retorno aos acionistas.

Existem diversos tipos de riscos a qual uma Instituição financeira esta exposta, o novo acordo de capital da Basiléia (Basileia
II), aborda três riscos principais, que são: Risco de Crédito, Risco de Mercado e Risco Operacional. O controle destes riscos com o acordo de Basiléia trouxe maior solidez ao sistema financeiro mundial.

Devido às características operacionais das Instituições financeiras, o risco de crédito pode ser considerado como o principal tipo de risco a ser gerenciado. $\mathrm{O}$ risco de crédito pode ser definido como a impossibilidade do tomador dos empréstimos não honrar com a sua obrigação na data futura, o que pode ser gerado tanto por incapacidade de pagamento como por descumprimento do prazo de pagamento (Gestel e Baesens 2009).

De acordo com Treacy e Carey (2000, p. 171) "O risco de crédito de um empréstimo ou outra exposição durante um determinado período envolve tanto a probabilidade de default (PD) como a fração dos empréstimos que é suscetível de perda em caso de inadimplência (LIED)", o produto entre (PD) e (LIED) é a taxa de perda esperada da exposição (LE).

Outras definições para risco de crédito podem ser encontradas na literatura. Para Lopez e Saidenberg (2000. p. 152) "Risco de crédito pode ser definido como o grau de flutuação do valor em instrumentos de dividas e derivativos, devido à mudança na qualidade de crédito subjacente dos mutuários e contraparte".

A importância da medição do risco de credito evoluiu imensamente nas ultimas duas décadas, fazendo com que a medida do risco de crédito tenha se tornado extremamente importante, entre os fatores que levaram as medidas de risco como uma ferramenta essencial para as Instituições financeiras, está o aumento do numero de falências, margens mais competitivas na concessão de empréstimos e o aumento da exposição do risco de inadimplência (Altman e Saunders, 1998). 
A gestão de risco de crédito envolve a medição do risco de crédito. A medida geralmente utilizada para medir o risco são o Credit Scoring e o Credit Rating, a diferença entre as duas metodologias de medição, na visão de (Gestel e Baesens 2009, p. 117) "acabou ficando distorcida".

Os modelos de Credit scoring e Credit Rating, de acordo com Gestel et al (2009, p. 92 ) é uma "Técnica de gestão de risco do tomador do empréstimo, a pontuação de crédito atribui a cada cliente um nível de risco". Tal medida busca separar o bom pagador do mau pagador, diminuindo assim o risco do não recebimento dos empréstimos concedidos.

Durand (1941) afirma que as fórmulas para a classificação de crédito são geralmente compostas pela combinação de experiência e intuição. $\mathrm{O}$ autor fez uso da análise discriminante múltipla para analise de crédito, o trabalho de Duran, é considerado com um dos mais antigos a utilizar essa metodologia para analise do risco de crédito. O autor fez uso de métodos estatísticos para criar uma formula de Credit Rating.

Outro estudo de grande importância no que se refere a analise de crédito foi realizado por Altman (1968), o autor fez uso da metodologia de analise discriminante múltipla para a previsão de falência.

Altman (1968) utilizou um conjunto de índices financeiros e econômicos para realização do estudo, dando foco as empresas de manufatura. O resultado desse trabalho foi à criação do Z-Score, o autor conclui que o modelo se mostrou extremamente preciso, prevendo corretamente $94 \%$ da previsão de falência da amostra inicial, sendo que a amostra inicial foi composta por 66 empresas divididas em dois grupos, o grupo de empresas que decretaram falência e o outro grupo consistiu de um grupo de empresas escolhidas aleatoriamente.
Além das metodologias utilizadas por Duram (1941) e Altman (1968) é possível verificar na literatura acadêmica, outras metodologias estatísticas utilizadas para o desenvolvimento de modelos de classificação de crédito.

Thomas (2000) apresenta um estudo que analisa as técnicas utilizadas nos modelos de risco de crédito, por meio do trabalho o autor procura discutir a necessidade de incorporar as condições econômicas nos sistemas de credit scoring. No estudo o autor identificou que as decisões para concessão de crédito baseavam-se somente nos $5 \mathrm{cs}$ do crédito, evoluindo nos dias de hoje para métodos estatísticos. Entre os modelos estatísticos utilizados o autor menciona a analise discriminante como método mais utilizado, porem ressalta que a analise discriminante é criticada por ser eficiente apenas para pequenas classes de distribuições. $\mathrm{O}$ autor também menciona a analise de regressão sendo utilizada em desenvolvimento de modelos de medição crédito. A regressão logística, a análise Probit e redes neurais também são mencionadas pelo autor com técnicas utilizado pelas instituições como métodos estatísticos para desenvolvimento de modelos para medir o risco de crédito.

Abdou, Masry e Pointon, (2007) indo ao encontro de Thomas (2000) mencionam que é possível destacar a analise discriminante, analise de regressão, analise Probit e regressão logística. (Abdou, Masry e Pointon, 2007).

Lopez e Saidenberg (2000) propõem um método de avaliação de modelos de risco usando abordagem de dados em Painel. Os autores mencionam a importância para os bancos e para as agências reguladoras, de se avaliar a precisão dos modelos utilizados atualmente para prever as perdas relacionadas ao crédito, foi proposto no artigo uma avaliação para os modelos de risco de crédito 
usando cross-section, avaliando os modelos não só sobre o tempo, mas também para prever o risco relacionado ao crédito em diversos pontos no tempo. Os autores concluem que o horizonte de tempo geralmente utilizado no modelo de crédito prejudica a previsão de inadimplência, a pesquisa menciona que o método proposto no estudo precisa de pesquisas adicionais.

Outros estudos também foram realizados para testar a efetividade dos modelos de crédito utilizado pelas instituições financeiras, para verificar a eficiência dos modelos de classificação de credito utilizado para separar o bom pagador do mau pagador. Treacy e Carey (2000) apresentam um estudo que descreve os sistemas de rating de crédito das 50 maiores instituições financeiras dos Estados Unidos. Os autores procuram analisar a efetividade dos sistemas internos de Rating utilizados pelas instituições financeiras. Com os estudos, os autores chegaram à conclusão que certas características dos atuais sistemas de classificação de crédito devem ser revistos, trazendo assim melhor gerenciamento do risco pela instituição e a obtenção de vantagens das oportunidades que possam surgir.

Os autores ainda fornecem alguns aconselhamentos para os bancos e para as agências reguladoras, entre eles, vale destacar o aconselhamento dado aos bancos, onde o autor afirma que os bancos devem fazer tentativas sérias para melhor quantificar as características das perdas, não deixando a tarefa apenas nas mãos dos especialistas em gestão de risco, mas também envolver todos os stakeholders.

Jacobson e Roszbach (2003) apresentam um método para avaliar os modelos de calculo do risco de crédito de uma carteira, os autores afirmam que os modelos de Credit Scoring sofrem de um viés de seleção

Augusto Guzzo Revista Acadêmica, 2013, № 12, 57-66 amostral e sugerem um modelo de scoring imparcial utilizando Probit bivariado.

O conjunto de dados do estudo analisou 13.338 pedidos de empréstimos na principal Instituição fornecedora de empréstimos da Suécia, entre o período de Setembro de 1994 a Agosto de 1995.

Por meio de uma seleção eficiente, pode reduzir o risco de crédito em até $80 \%$. Com o estudo, os autores chegaram à conclusão que o banco concedia empréstimos com base em modelos desatualizados e de uma maneira não consistente para a minimização do default de crédito. Os autores ainda afirmam que o calculo do $\mathrm{VaR}$ por meio de uma seleção eficiente, pode reduzir o risco de crédito em até $80 \%$.

A concessão de crédito pode ser descrita como uma das principais ferramentas para o desenvolvimento do econômico de um país. (Jacobson e Roszbach, 2003) Segundo o Banco Central (BC) a média diária de concessão de crédito tem sofrido um acréscimo de $8,1 \%$, sendo que as concessões de crédito fornecidas pelos bancos para pessoa jurídica cresceram $11 \%$ em relação ao mês de Julho para Agosto de 2012.

Diante de um cenário do aumento constante na concessão de crédito, e da evolução dos modelos de medição do risco de crédito nas ultimas décadas, surge à necessidade de metodologias eficazes de avaliação do risco de crédito, tais necessidade surgem principalmente do aumento do numero de falências. (Altman, Sunders, 1998).

Com base nos trabalhos analisados no referencial teórico, foi possível perceber a necessidade de avaliação dos modelos de risco de crédito, tanto por parte das Instituições financeiras como por parte dos agentes reguladores.

Com este estudo pretende-se contribuir para o gerenciamento do risco de crédito nas 
Instituições financeiras Brasileira, caracterizado por um constante aumento da exposição a esse tipo de risco.

\section{Metodologia}

A metodologia utilizada neste trabalho foi qualitativa, com a utilização de diversos conceitos relativos à gestão de risco de crédito, com base em referências internacionais. A pesquisa realizada é de caráter descritivo-exploratório, que segundo Deslauriers et al (2008) pode ser utilizada com dois objetivos: conhecer para mudar ou conhecer para aprofundar.

No caso deste estudo, procura-se entender para aprofundar, tendo como objetivo verificar a fragilidade das empresas financeiras brasileiras diante de um cenário de crise de crédito.

A amostra deste estudo é composta por instituições financeiras brasileiras de capital aberto, listadas na Bolsa de Valores de São
Paulo (BOVESPA). A base de dados foi composta por relatórios de Gestão de Risco disponibilizado pelas Instituições Financeiras Brasileiras através do relatório de risco e por meio deles foi possível verificar os modelos de gestão de risco de crédito utilizados pelas empresas.

Com base no referencial teórico apresentado, optou-se por fazer um comparativo entre os modelos de risco de crédito apresentados pela literatura e os modelos de risco de crédito utilizado pelas instituições financeiras brasileiras.

\section{Análise dos Resultados}

O Quadro 1, apresenta as principais instituições financeira listadas na Bolsa de Valores de São Paulo (BOVESPA), bem como o segmento de governança corporativa, o modelo de mensuração de risco de crédito utilizados e outros métodos utilizados na concessão de crédito.

Quadro 1 - Principais Instituições Financeiras de Capital Aberto no Brasil.

\begin{tabular}{|l|l|l|}
\multicolumn{1}{|c|}{ Razão Social } & \multicolumn{1}{|c|}{ Modelo utilizado } & \multicolumn{1}{|c|}{$\begin{array}{c}\text { Outros Métodos utilizados } \\
\text { na concessão de crédito }\end{array}$} \\
\hline ABC BRASIL & Credit Scoring e Credit Rating & Análise gerencial \\
\hline ALFA CONSORC & Credit Scoring e Credit Rating & Análise gerencial \\
\hline AMAZONIA & Credit Scoring e Credit Rating & Garantias \\
\hline BANESE & Credit Scoring e Credit Rating & Garantias e análise do sistema \\
\hline BANESTES & Credit Scoring e Credit Rating & Garantias e provisão \\
\hline BANPARA & Não divulgado & Análise gerencial \\
\hline BANRISUL & $\begin{array}{l}\text { Behaviour Score, Credit Rating e Credit } \\
\text { Scoring }\end{array}$ & Análise de comitê de crédito \\
\hline BICBANCO & Credit scoring & Garantias \\
\hline BRADESCO & $\begin{array}{l}\text { Behaviour Score, Credit Rating e Credit } \\
\text { Scoring. }\end{array}$ & Garantias e comitê de crédito \\
\hline BRASIL & Credit Scoring e Credit Rating & $\begin{array}{l}\text { Frequência Esperada de } \\
\text { inadimplência (FEI) }\end{array}$ \\
\hline BRB BANCO & Credit Scoring & Análise de comitê de crédito \\
\hline
\end{tabular}




\begin{tabular}{|l|l|l|} 
BTGP BANCO & $\begin{array}{l}\text { Probabilidade de Default (PD) e Credit } \\
\text { rating }\end{array}$ & $\begin{array}{l}\text { Garantia e Análise de comitê de } \\
\text { crédito }\end{array}$ \\
\hline DAYCOVAL & Não divulgado & Análise de comitê de crédito \\
\hline INDUSVAL & $\begin{array}{l}\text { Credit Rating estabelecido pela gerencia e } \\
\text { não por modelos }\end{array}$ & Análise de comitê de crédito \\
\hline ITAUUNIBANCO & $\begin{array}{l}\text { Probabilidade de Default (PD), Credit } \\
\text { rating e Credit Scoring }\end{array}$ & Garantias e comitê de crédito \\
\hline MERC BRASIL & Sistema de Credit Risk Intelligence - CRI & Garantias \\
\hline PANAMERICANO & Credit Scoring e Credit Rating & Análise gerencial \\
\hline SANTANDER BR & Credit Scoring & Análise de comitê de risco \\
\hline
\end{tabular}

Fonte: Elaborado pelo autor com base nos relatórios de gestão de risco das empresas financeiras listadas em bolsa.

Com base na análise dos relatórios de riscos das instituições financeiras brasileiras foi possível constatar a preferência pela utilização de modelos de Credit Scoring e Credit Rating para mitigar de risco de crédito (gráfico 1). Dentre as 18 empresas analisadas, 16 empresas utilizam os modelos de Credit Rating e Credit Scoring para realizar a concessão de empréstimos. Também foi possível constatar que empresas de porte maior utilizam outras metodologias adicionais os modelos de Credit Scoring e Credit Rating, como é o caso de ItauUnibanco e BTG, que utilizam outros modelos de crédito para mitigar o risco de inadimplência.

Gráfico 1 - Modelos de análise de concessão de crédito utilizados pelas Instituições financeiras de capital aberto no Brasil.

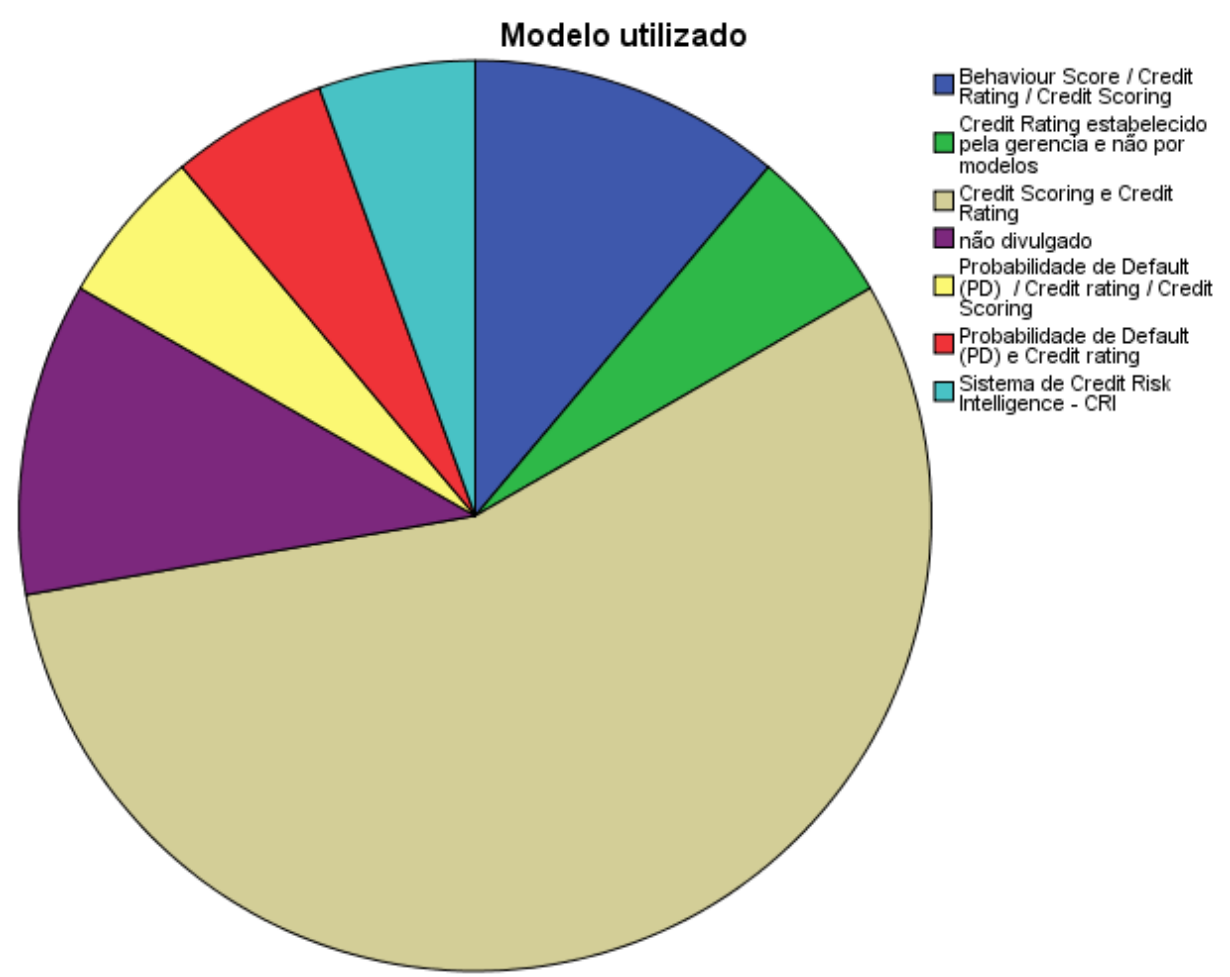

Fonte: Elaborado pelo autor com base nos relatórios de gestão de risco das empresas financeiras lista em bolsa 
Outro ponto de destaque na análise é a utilização de comitês de crédito, que em complemento aos modelos de gestão de risco, atuam de forma a mitigar o risco de inadimplência. De acordo com o gráfico 2, das 18 empresas financeiras analisadas, 13 delas possuem um comitê de analise de crédito, que são responsáveis pelo parecer final de concessão ou não do empréstimo. Também vale destacar a exigência de garantias vinculadas aos empréstimos, é possível verificar que é uma prática comum dentre os grandes bancos brasileiros a exigência de garantias, que agem como um complemento aos modelos de risco de crédito e analise dos comitês de crédito.

Gráfico 2 - Modelos de análise de concessão de crédito utilizados pelas Instituições financeiras de capital aberto no Brasil.

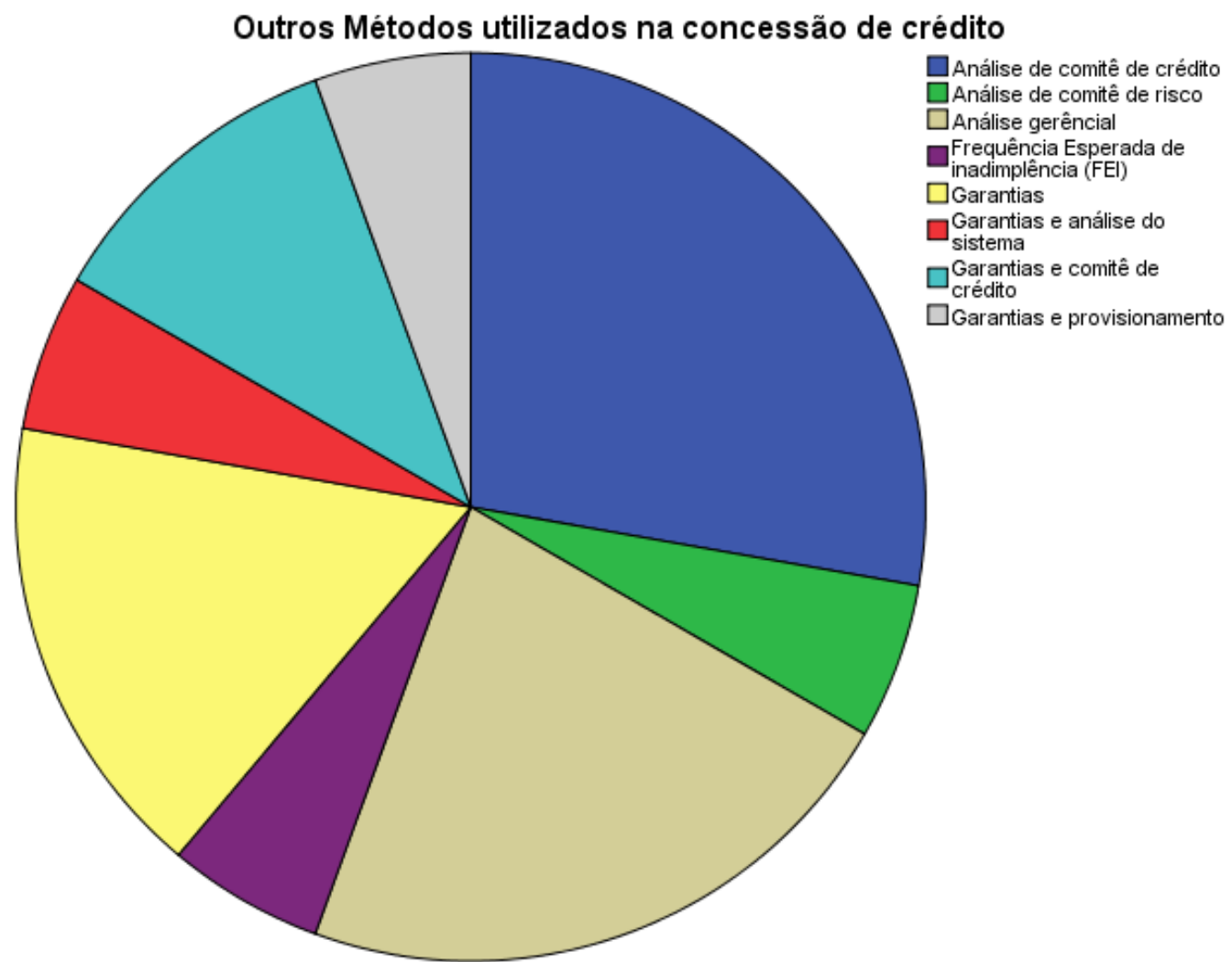

Fonte: Elaborado pelo autor com base nos relatórios de gestão de risco das empresas financeiras lista em bolsa

\section{Conclusão}

O objetivo deste trabalho foi analisar as estratégias utilizadas pelas instituições financeiras brasileiras no gerenciamento do risco de crédito. Fazendo uso dos relatórios de gerenciamento de risco destas instituições, buscou-se identificar as medidas implantadas para a diminuição da exposição a esta modalidade de risco.

Ao se realizar a análise dos relatórios de risco das instituições financeira, buscaram-se identificar há existência de similaridades entre o verificado na literatura e o colocado em prática pelos Bancos Brasileiros. 
Como resultado das análises, destaca-se a preferência pela utilização dos modelos de Credit Scoring e Credit Rating que de acordo com a constatação de Jacobson e Roszbach (2003) sofrem de um viés de seleção amostral, podendo prejudicar a eficácia da previsão da inadimplência.

Diante do aumento da importância dos modelos de medição do risco de crédito é essencial avaliar os modelos utilizados pelas instituições financeiras Brasileira, a fim de verificar se os modelos são os mais adequados. De acordo com Lopez e Saidenberg (2000) existem diversos modelos de risco de crédito, variando em diversos níveis de sofisticação.

Por fim, os modelos de gerenciamento de risco de crédito em conjunto com as garantias ou análise de comitê de crédito, pode diminuir o risco de inadimplência, dando mais solidez ao mercado financeiro brasileiro.

\section{Referências Bibliográficas}

1. ALTMAN, E.I. (1968). Financial Ratios, Discriminant Analysis and the Prediction of Corporate Bankruptcy. The Journal of Finance, XXIII: 589-609.

2. ALTMAN, E. I.; SAUNDERS, A. (1998) Credit risk measurement: Developments over the last 20 years - Journal of Banking \& Finance 21 - p. 1721 - 1742.

3. Agencia de classificação de Rating Austin Rating - Prejuízo de bancos com calotes

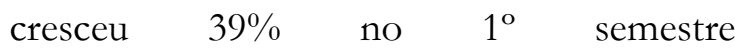
<http://www.austin.com.br/Imprensa> Acessado em 27/11/2012.
4. Banco Central do Brasil (BC). Relatório de Economia Bancária e Crédito <http://www.bcb.gov.br/Pec/spread/port /relatorio_economia_bancaria_credito.pdf> acessado em 28/11/2012.

5. Banco Central do Brasil (BC). Basiléia $2<$ http://www.bcb.gov.br/?BASILEIA2> acessado em 29/11/2012.

6. DESLAURIERS, J.P. \& KÉRISIT, M. O delineamento de pesquisa qualitativa. In: Apesquisa qualitativa: enfoques epistemológicos e metodológicos. Tradução de Ana Cristina Nasser. Petrópolis, RJ: Vozes, 2008. pp. 127-153.

7. DURAND, D. (1941). Risk Elements in Consumer Instalment Financing, Studies in Consumer Instalment Financing. New York: National Bureau of Economic Research.

8. DHAR, S. e BALAKRISHNAN, B. (2006) Risks, Benefits, and Challenges in Global IT Outsourcing: Perspectives and Practices. Journal of Global Information Management, vol. 14 , issue 3 .

9. Federal Deposit Insurance Corporation FDIC. Failed Banklist < www.fdic.gov/bank/individual/failed/banklist.html.>

Acessado em 02/09/2013.

10. FROOT, K. A.; SCHARFSTEIN, D. S.; STEIN, J. C. (1993) Risk Management: Coordinating Corporate Investments and Financing Policies. The Journal of Finance, v. XLVIII, n. 5, p. 1629-1658. 
11. GESTEL, T. V.; BART B. (2009) Credit Risk Management: Basic concepts: Financial risk components, Rating analysis, models, economic and regulatory capital. Oxford.

12. HANSSON, S. O. (2005) Seven Myths of Risk. Risk Management, Vol. 7, No. 2.

13. JACOBSON, T; ROSZBACH K. (2003)

Bank lending policy, credit scoring and valueat-risk - Journal of Banking \& Finance 27, p. 615-633.

14. KAPLAN, S. e GARRICK, B.J. (1981)

On the quantitative definition of risk. Risk Analysis, 1, 11-27.

15. LOPEZ J. A; SAIDENBERG M. R. (2000) - Evaluating credit risk models Journal of Banking \& Finance 24 151-165.

16. POLAK, P; ROBERTSON, D. C.; LIND, M. (2011) The New Role of the Corporate Treasurer: Emerging Trends in
Response to the Financial Crisis. International Research Journal of Finance and Economics. Euro Journals Publishing, Inc.

17. RAWLS, S. W.; SMITHSON, C. (1990) Strategic risk management, Continental Bank Journal of Applied Corporate Finance 1, 618.

18. STULZ R. M. - (1996) Rethinking risk management - Bank of America Journal of Apllied Corporate Finance.

19. THOMAS L. C. (2000) - A survey of credit and behavioural scoring: forecasting financial risk of lending to consumers International Journal of Forecasting 16 - p. 149-172

20. TREACY, W. F; CAREY, M. (2000) Credit risk rating systems at large US banks Journal of Banking \& Finance 24, p. 167-201. 